\title{
Concurrent chemoradiotherapy with nedaplatin in patients with stage IIA to IVA cervical carcinoma
}

\author{
TORU FUJIOKA, TOSHIAKI YASUOKA, MASAE KOIZUMI, HIROKI TANAKA, \\ HISASHI HASHIMOTO, MOTOO NABETA, KOJI KOIZUMI, YUKO MATSUBARA, \\ KATSUYUKI HAMADA, KEIICHI MATSUBARA, TOMIHIRO KATAYAMA and AKIHIRO NAWA \\ Department of Obstetrics and Gynecology, Ehime University, Graduate School of Medicine, Ehime, Japan
}

Received July 8, 2012; Accepted September 14, 2012

DOI: $10.3892 / \mathrm{mco} .2012 .27$

\begin{abstract}
The present study aimed to evaluate the efficacy and toxicities of nadaplatin-based concurrent chemoradiotherapy (CCRT) in patients with stage IIA to IVA cervical carcinoma. Patients with an International Federation of Gynecology and Obstetrics (FIGO) stage IIA to IVA cervical carcinoma were treated with nadaplatin-based CCRT, using high-dose rate intracavitary brachytherapy (HDR-ICBT) or radiotherapy (RT) alone, in patients with FIGO stage IIA to IVA cervical carcinoma. CCRT with nedaplatin $\left(80 \mathrm{mg} / \mathrm{m}^{2}\right)$ was administered on Days 1 and 29. The records of 17 women treated either with nadaplatin-based CCRT using HSR-ICBT $(n=8)$ or RT alone ( $\mathrm{n}=9$ ), for stage IIA to IVA cervical carcinoma were retrospectively reviewed. The activity and toxicity were compared in the two treatment groups. Progression-free survival (PFS) and overall survival (OS) were the main endpoints. The 5-year overall survival rates in the CCRT and RT groups were 68.6 and $77.8 \%$, respectively. The median OS of the CCRT and RT groups was 38.5 and 27.3 months, respectively. There was no significant difference in either PFS $(\mathrm{P}=0.618)$ or OS $(\mathrm{P}=0.231)$. The most common grade $3-4$ or higher toxicities in the CCRT groups were leuko-/neutropenia (37.5\%). The frequency of acute grade 3-4 toxicity was higher in the CCRT compared to the RT group. However, no statistically significant difference was observed. Nedaplatin-based CCRT was safely performed. Although the prognosis of patients with FIGO stage IIA to IVA cervical carcinoma was not significantly improved, fewer distant relapses were observed in this treatment. Consequently, nedaplatin-based CCRT may be considered as a potential alternative to cisplatin-based CCRT in this patient population.
\end{abstract}

Correspondence to: Dr Toru Fujioka, Department of Obstetrics and Gynecology, Ehime University, Graduate School of Medicine, Shitsukawa, Toon, Ehime 791-0295, Japan

E-mail: fujioka@m.ehime-u.ac.jp

Key words: nedaplatin, cervical carcinoma, concurrent chemoradiotherapy

\section{Introduction}

Cervical carcinoma is the second most common cause of carcinoma worldwide (1). Radiotherapy (RT) is the major treatment modality for invasive cervical carcinomas; however, the prognosis is poor in advanced uterine cervical carcinoma $(2,3)$.

In the 1990s, numerous attempts were made to improve the prognosis for advanced uterine cervical carcinoma by concurrently using radiotherapy and chemotherapy (CCRT). These attempts led to the results of five randomized controlled trials demonstrating that CCRT was effective against advanced uterine cervical carcinoma, while decreasing the risk of mortality thereof by $30-50 \%$, announced by the National Cancer Institute (USA) in February 1999 (4-8). However, data from these clinical trials demonstrated several problems when this CCRT was employed in Japanese patients. Some of the questions addressed were whether or not the reported dose of cisplatin was appropriate for Japanese women, whether the use of cisplatin was appropriate for patients with advanced uterine cervical carcinoma given their possibly reduced renal function, or to what extent platinum-containing drugs are likely to be effective in such patients in the place of cisplatin.

Nedaplatin (cis-diammine-glycoplatinum), a platinum analog, was developed by the pharmaceutical company Shionogi \& Co., Ltd. (Tokushima, Japan), aiming to produce a treatment with lower renal and gastrointestinal toxicity that is as effective as cisplatin. Clinically, previous phase II studies conducted in Japan suggested that nedaplatin had particularly strong clinical efficacy on a squamous cell carcinoma of the head and neck, esophagus and uterine cervix $(9,10)$. In a phase II clinical trial, nedaplatin demonstrated a $46 \%$ response rate in patients with recurrent cervical carcinoma, which was slightly superior compared to cisplatin (39\%) (11).

Its lower incidence of nephrotoxicity in comparison with cisplatin was demonstrated to be associated with a difference in the kidney distribution of these drugs. When the two agents were administered at the same dose, the accumulation of nedaplatin in the kidney was $40 \%$ compared to cisplatin, leading to a lower nephrotoxicity of nedaplatin $(12,13)$. Since nedaplatin exhibited minimal nephrotoxicity, it may be used in patients with marginal renal function $(14,15)$.

The radiosensitizing properties of nedaplatin have been demonstrated in several preclinical studies $(16,17)$. However, 
the use of nedaplatin in the clinical setting of CCRT in patients with cervical carcinoma is limited, CCRT with the administration of nedaplatin at $80 \mathrm{mg} / \mathrm{m}^{2}$ on Days 1 and 29 has been reported to be safe and effective (18).

The present is a retrospective study conducted to evaluate whether or not nedaplatin-based CCRT is safe and superior to RT alone in Japanese patients with advanced stage of cervical carcinoma.

\section{Materials and methods}

Patients. Patients with confirmedFIGO stage IIA to IVAcervical carcinoma, who had been treated between 2006 and 2010 in the Ehime University Hospital were eligible. No previous chemotherapy or RT was allowed. Other eligibility criteria included age (20-80 years) and performance status (score $0-2$ ).

The patients had primary, previously untreated and histologically confirmed carcinoma of the uterine cervix. The pretreatment workup comprised a complete medical history and physical examination, complete blood count, biochemistry panels, chest X-ray and computed tomography (CT) of the abdomen and pelvis, magnetic resonance imaging (MRI) and optional intravenous pyelography and cystoscopy. Regarding lymph nodes measuring $\geq 10 \mathrm{~mm}$ along their longest axis on CT or MRI were defined as metastatic nodes. Patients with radiologic evidence of para-aortic disease were excluded. MRI was used to evaluate the size and geometry of the primary tumor. The maximal tumor diameter was measured on T2-weighted images. The longest diameter was considered to be the maximal tumor diameter.

Patients were staged clinically by both a gynecological oncologist and a radiation oncologist, according to the International Federation of Gynecology and Obstetrics staging criteria, without general anesthesia. Patients, who received extended-field RT were also excluded.

Radiotherapy. Patients were treated with conventional radiotherapy consisting of external beam radiotherapy (EBRT) and high-dose rate intracavitary brachytherapy (HDR-ICBT). The EBRT was performed using a 10 megavolt (MV) X-ray from a linear accelerator. Using anteroposterior parallel opposed portals, the external irradiation was delivered to the whole pelvis at $2 \mathrm{~Gy} /$ fraction for 5 fractions/week, for a total of 25 fractions (50 Gy). The superior margin of the external radiation field was placed on the upper border of the fifth lumbar vertebra, while the inferior margin was the inferior border of the obturator foramen, although this margin extended inferiorly when there was vaginal invasion. Laterally, the field extended $1.5-2 \mathrm{~cm}$ beyond the lateral margin of the bony pelvic wall. A midline block ( $4 \mathrm{~cm}$ width at the midline) was inserted into the central lower two-thirds length of the pelvic field after 30 Gy had been delivered.

Subsequent to adequate tumor regression, the HDR-ICBT was performed once a week during the course of the EBRT with the centrally shielded field. Usually, the first HDR-ICBT was applied after 30 Gy of EBRT. ICBT was administered to the patients using a microselectron HDR. In patients with vaginal infiltration or with a narrow vagina, a tandem with a vaginal cylinder was used. The ICBT dose was delivered to point $\mathrm{A}$, defined as $2 \mathrm{~cm}$ above the cervical $\mathrm{OS}$ marker and
Table I. Patient characteristics.

\begin{tabular}{|c|c|c|c|}
\hline Characteristic & $\begin{array}{c}\text { CCRT-group } \\
(\%)\end{array}$ & $\begin{array}{c}\text { RT-group } \\
(\%)\end{array}$ & P-value \\
\hline No. of patients & 8 & 9 & \\
\hline \multicolumn{4}{|l|}{ Age (years) } \\
\hline Median & 63.5 & 74.1 & 0.0356 \\
\hline Range & $51-78$ & $58-79$ & \\
\hline \multicolumn{4}{|l|}{ Clinical stage } \\
\hline IIA & $0(0)$ & $3(33.3)$ & 0.1781 \\
\hline IIB & $2(25.0)$ & $0(0)$ & \\
\hline IIIA & $1(12.5)$ & $2(22.2)$ & \\
\hline IIIB & $4(50.0)$ & $4(44.4)$ & \\
\hline IVA & $1(12.5)$ & $0(0)$ & \\
\hline \multicolumn{4}{|l|}{ Histology } \\
\hline Squamous cell & $6(75.0)$ & $9(10)$ & 0.2794 \\
\hline Adenocarcinoma & $1(12.5)$ & 0 & \\
\hline Adenosquamous & $1(12.5)$ & 0 & \\
\hline \multicolumn{4}{|c|}{ Tumor diameter (mm) } \\
\hline Median & 47.5 & 60.0 & 0.0700 \\
\hline Range & $44-87$ & $54-86$ & \\
\hline \multicolumn{4}{|c|}{$\begin{array}{l}\text { Pretreatment } \\
\text { hemoglobin level }(\mathrm{g} / \mathrm{dl})\end{array}$} \\
\hline Median & 11.5 & 11.1 & 0.6583 \\
\hline Range & $9.3-14.8$ & $8.0-12.8$ & \\
\hline \multicolumn{4}{|l|}{$\begin{array}{l}\text { Pretreatment } \\
\text { SCCA level (ng/ml) }\end{array}$} \\
\hline Median & 7.7 & 34.2 & 0.1034 \\
\hline Range & $1.1-51.6$ & $0.8-250$ & \\
\hline
\end{tabular}

CCRT, concurrent chemoradiotherapy; SCCA, squamous cell carcinoma antigen; RT, radiotherapy.

$2 \mathrm{~cm}$ perpendicular to the uterine axis, along the plane of the uterus. The planned total dose of HDR-ICBT was 24 Gy in 4 fractions. No EBRT was performed on the same day as HDR-ICBT (Fig. 1).

Chemotherapy. Chemotherapy was administered intravenously with nedaplatin during the course of EBRT on Days 1 and 29, but was not administered on the same day as ICBT. The dose of nedaplatin was $80 \mathrm{mg} / \mathrm{m}^{2}$ (range, 60-80). The drug was administered in a 2-h infusion. Renal function and blood counts were assessed prior to each cycle. Nedaplatin administration was suspended when the granulocyte count was $<1,500 / \mu 1$ or the platelet count was $<100,000 / \mu 1$. During the weeks the patient did not receive chemotherapy, radiation was continued, provided the white blood cell count was $>2,000 / \mu 1$ and the platelet count was $>50,000 / \mu 1$.

Control patients. A non-randomized group of patients with stage IIA to IVA cervical carcinoma treated with definitive RT alone between 2006 and 2010 served as the control group. RT for these patients consisted of a combination of EBRT (50 Gy 
Table II. Treatment and survival.

\begin{tabular}{|c|c|c|c|}
\hline Variables & $\begin{array}{l}\text { CCRT-group } \\
\quad(n=8)\end{array}$ & $\begin{array}{l}\text { RT-group } \\
\quad(n=9)\end{array}$ & P-value \\
\hline \multicolumn{4}{|c|}{$\begin{array}{l}\text { Dose of nedaplatin } \\
\text { administered }\left(\mathrm{mg} / \mathrm{m}^{2}\right)\end{array}$} \\
\hline Median & 75.0 & - & \\
\hline Range & $60-80$ & - & \\
\hline \multicolumn{4}{|c|}{ Duration of RT (days) } \\
\hline Median & 50.9 & 49.0 & 0.7677 \\
\hline Range & $44-61$ & $41-61$ & \\
\hline \multicolumn{4}{|c|}{$\begin{array}{l}\text { Patients with } \\
\text { recurrence }(\%)\end{array}$} \\
\hline Local & 2 & 0 & 0.1428 \\
\hline Distant & 1 & 4 & \\
\hline \multicolumn{4}{|c|}{ Initial response } \\
\hline $\mathrm{CR}$ & 7 & 9 & 0.4706 \\
\hline PR & 1 & 0 & \\
\hline \multicolumn{4}{|c|}{ PFS (months) } \\
\hline Median & 30.5 & 27.3 & 0.6182 \\
\hline Range & $4-63$ & $6-61$ & \\
\hline Mean & 33.6 & 28.5 & \\
\hline \multicolumn{4}{|c|}{ OS (months) } \\
\hline Median & 38.5 & 27.3 & 0.2312 \\
\hline Range & $17-67$ & $10-63$ & \\
\hline Mean & 41.5 & 29.9 & \\
\hline
\end{tabular}

CCRT, concurrent chemoradiotherapy; RT, radiotherapy; CR, complete response; PR, partial response; PFS, progression-free survival; OS, overall survival.

to the whole pelvis) and HDR-ICBT (24 Gy to point A), which was the same as the treatment for the CCRT-treated patients.

Toxicity. Clinical data regarding treatment-related complications were also collected. Complications that occurred within 90 days subsequent to the initiation of primary treatment were considered to be acute complications. The severity of acute complications was classified, according to the National Cancer Institute Common Terminology Criteria for Adverse Events, version 2.0 .

Follow-up. During the treatment, the patients were evaluated weekly by pelvic examination and complete blood count. In patients treated with chemoradiation, renal and liver function tests were also performed on a weekly basis. Patients were monitored regularly and examined for acute toxicity by both gynecological and radiation oncologists. Subsequent to termination of the treatment, patient follow-up occurred in an outpatient clinic on a monthly basis during the first year, bimonthly during the second year, every 3 months during the third year, every 6 months during the fourth to fifth years and annually thereafter. Follow-up procedures included gynecological examination, cervical cytology and squamous cell

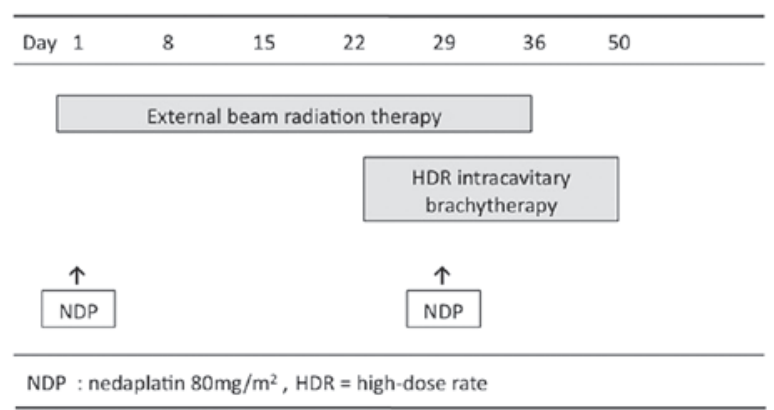

Figure 1. Treatment schedule for the 8 concurrent chemoradiotherapy (CCRT)-treated patients.

carcinoma antigen (SCCA) evaluation. Evaluation with CT was repeated every 6 months during the first 2 years and once a year thereafter. Pelvic failure, including central and parametrial failure, was defined as persisting or recurring disease in the pelvis. When pelvic examination or smears detected a potential local recurrence, a biopsy was taken for confirmation whenever possible. Distant failure was defined as a disease occurring outside the pelvis, including the para-aortic lymph nodes. No patient was lost to follow-up. The median duration of the follow-up was 27.3 months (range, 10-67).

Statistical analysis. The differences between the groups with respect to stage, histology, the site of recurrence and treatment-related toxicity were assessed using Fisher's exact test. The maximum tumor diameter, pretreatment hemoglobin and SCCA levels were compared using Welch's t-test. The overall survival (OS) and progression-free survival (PFS) curves were calculated according to the Kaplan-Meier method. $\mathrm{P}<0.05$ was considered to indicate a statistically significant difference. Analyses were performed using the SPSS15.0 software.

\section{Results}

Patient characteristics. Between January, 2006 and December, 2010, 17 patients with FIGO stage IIA to IVA cervical carcinoma treated with nedaplatin-based CCRT $(n=8)$ or RT alone $(n=9)$ were identified. The clinicopathological characteristics of these patients are shown in Table I. The characteristics of the patients in the CCRT group were similar to those in the RT group. No significant differences were found among the patient characteristics, with the exception of age.

Treatment outcome. As shown in Table II, among the CCRT-treated patients, the median and mean PFS were 30.5 and 33.6 months, respectively, while the median and mean OS were 38.5 and 41.5 months, respectively. In CCRT-treated patients, the 5-year OS rate was $68.6 \%$. However, in the RT group, the median and mean PFS were 27.3 and 28.5 months, respectively, while the median and mean OS were 27.3 and 29.9 months, respectively (Table II). In RT-treated patients the 5-year OS rate was $77.8 \%$. When compared to the RT group, the CCRT group showed no statistically significant differences in terms of PFS (log-rank; $\mathrm{P}=0.5070)$ and $\mathrm{OS}$ (log-rank; $\mathrm{P}=0.5176$ ) (Fig. $2 \mathrm{~A}$ and $\mathrm{B}$ ). These findings indicate that the addition of concurrent nedaplatin to pelvic EBRT plus 
Table III. Patterns of failure.

\begin{tabular}{|c|c|c|c|}
\hline & \multicolumn{2}{|c|}{ Site of relapse, time until recurrence (month) } & \multirow[b]{2}{*}{ P-value } \\
\hline & CCRT-group $(\mathrm{n}=8)$ & RT-group $(n=9)$ & \\
\hline \multirow{3}{*}{$\begin{array}{l}\text { No. patients with recurrence } \\
\text { Type } \\
\text { Pelvic }\end{array}$} & $3(37.5 \%)$ & $4(44.4 \%)$ & 0.5806 \\
\hline & & & \\
\hline & $\begin{array}{l}1 \text { uterus, } 4 \\
1 \text { uterus, PLN, } 12\end{array}$ & 0 & 0.1428 \\
\hline \multirow[t]{2}{*}{ Distant } & & & \\
\hline & $1 \mathrm{PAN}, 38$ & $\begin{array}{l}1 \text { PAN, bone, } 5 \\
1 \text { liver, } 6 \\
1 \text { lung, } 9 \\
1 \text { PAN, } 14\end{array}$ & \\
\hline
\end{tabular}

CCRT, concurrent chemoradiotherapy; RT, radiotherapy; PLN, pelvic lymph node; PAN, para-aortic lymph node.

A

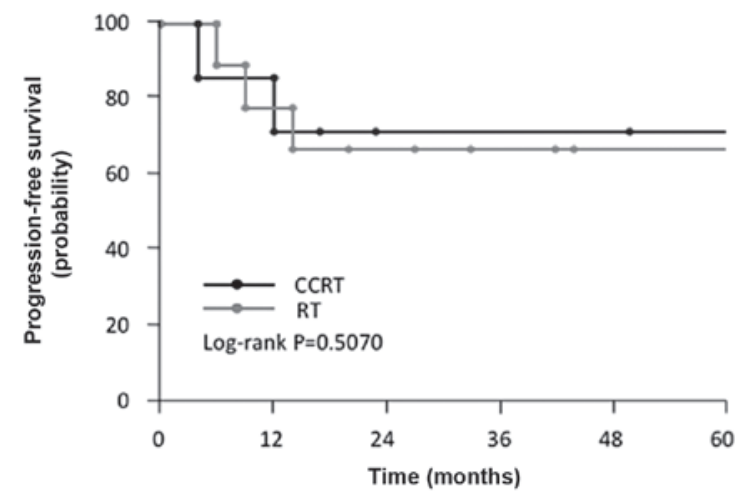

B

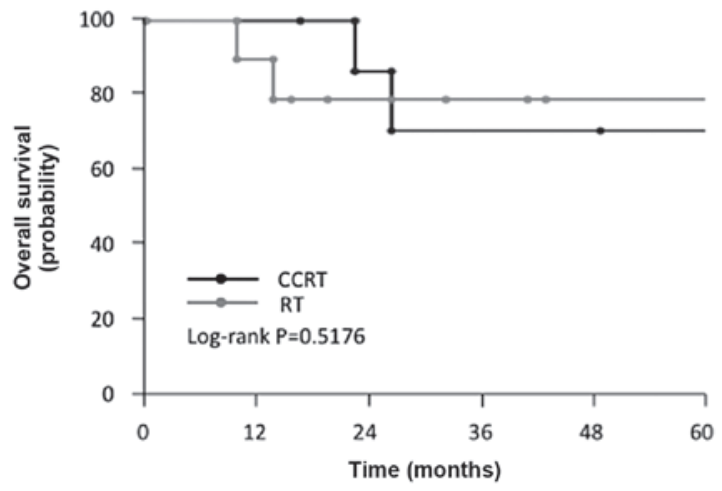

Figure 2. (A) Progression-free survival (PFS) among patients in the CCRT and RT groups is shown. PFS rate was higher in the CCRT group, with no statistically significant difference $(\mathrm{P}=0.5070)$. (B) Overall survival $(\mathrm{OS})$ in the CCRT and RT groups is shown. OS rate was higher in the CCRT group, with no statistically significant difference $(\mathrm{P}=0.5176)$.

HDR-ICBT did not significantly improve the prognosis in this patient population.

As shown in Table III, treatment failure was observed in 3 patients $(37.5 \%)$ in the CCRT group and in 4 patients $(44.4 \%)$ in the RT group. Of the 3 patients with treatment failures in the CCRT group, the first site of relapse was pelvic in 2 patients, while 1 patient developed distant relapse. The pelvic failures involved uterine recurrence. In the RT group, the 4 patients with treatment failure showed distant relapse. The distant site of relapse involved para-aortic lymph node, bone, lung and liver. The rate of distant relapse was higher in the RT compared to the CCRT group. However, the difference was not statistically significant $(\mathrm{P}=0.1428)$. No patient showed synchronous pelvic and distant relapse sites. The failure rate was not different in the two groups $(\mathrm{P}=0.5806)$.

Adverse effects. Generally, nedaplatin-based CCRT was well-tolerated. Among the patients in the CCRT group, the most frequently observed acute toxicity was hematologic. As shown in Table IV, in the CCRT group, grade 3 acute toxicity was observed in 3 patients $(37.5 \%)$. Of these patients, 1 had grade 3 neutropenia and required colony-simulating growth factor for 2 days. There were no grade 3-4 non-hematologic toxicities in either group.

In the RT group, although grade 1-2 acute toxicities, such as hematologic or gastrointestinal toxicity were commonly observed, no grade 3-4 non-hematologic toxicities were detected. The incidence of grade 3-4 acute toxicity was higher in the CCRT compared to the RT group. No other severe side effect was observed in either the CCRT or the RT group.

\section{Discussion}

CCRT, usually involving weekly cisplatin (CDDP) $\left(40 \mathrm{mg} / \mathrm{m}^{2}\right.$ for 6 weeks) in combination with RT, has been established as the standard treatment for locally advanced cervical carcinoma (4-8). However, CDDP has severe renal toxicity, thus heavy hydration is then required to undergo treatment-using CDDP. Nevertheless, nedaplatin is a derivative of cisplatin with lower renal toxicity, although similar to cisplatin.

Nedaplatin, an antineoplastic drug containing a platinum complex, was developed in order to provide superior antitumor effects to cisplatin and lower renal and gastrointestinal toxicity. Based on the results of a phase I clinical trial of nedaplatin, the drug was suggested to be administered as an intravenous infusion of $100 \mathrm{mg} / \mathrm{m}^{2}$ at 4 -week intervals (19). A phase II clinical trial using this dose regimen demonstrated a response 
Table IV. Acute toxicity.

\begin{tabular}{|c|c|c|c|c|c|}
\hline & $\begin{array}{c}\text { Grade } 0 \\
\mathrm{n},(\%)\end{array}$ & $\begin{array}{c}\text { Grade } 1 \\
\mathrm{n},(\%)\end{array}$ & $\begin{array}{c}\text { Grade } 2 \\
\mathrm{n},(\%)\end{array}$ & $\begin{array}{c}\text { Grade } 3 \\
\mathrm{n},(\%)\end{array}$ & $\begin{array}{c}\text { Grade } 4 \\
\mathrm{n},(\%)\end{array}$ \\
\hline \multicolumn{6}{|l|}{ CCRT-group } \\
\hline \multicolumn{6}{|l|}{ Hematologic } \\
\hline Leukopenia & $1(12.5)$ & $3(37.5)$ & $1(12.5)$ & $3(37.5)$ & $0(0.0)$ \\
\hline Neutropenia & $1(12.5)$ & $2(25.0)$ & $4(50.0)$ & $1(12.5)$ & $0(0.0)$ \\
\hline Anemia & $1(12.5)$ & $6(75.0)$ & $1(12.5)$ & $0(0.0)$ & $0(0.0)$ \\
\hline Thrombocytopenia & $8(100)$ & $0(0.0)$ & $0(0.0)$ & $0(0.0)$ & $0(0.0)$ \\
\hline \multicolumn{6}{|l|}{ Non-hematologic } \\
\hline Nausea/vomiting & $7(87.5)$ & $0(0.0)$ & $1(12.5)$ & $0(0.0)$ & $0(0.0)$ \\
\hline Diarrhea & $5(62.5)$ & $2(25.0)$ & $1(12.5)$ & $0(0.0)$ & $0(0.0)$ \\
\hline Radiation dermatitis & $7(87.5)$ & $1(12.5)$ & $0(0.0)$ & $0(0.0)$ & $0(0.0)$ \\
\hline \multicolumn{6}{|l|}{ RT-group } \\
\hline \multicolumn{6}{|l|}{ Hematologic } \\
\hline Leukopenia & $4(44.5)$ & $3(33.3)$ & $2(22.2)$ & $0(0.0)$ & $0(0.0)$ \\
\hline Neutropenia & $5(55.6)$ & $2(22.2)$ & $2(22.2)$ & $0(0.0)$ & $0(0.0)$ \\
\hline Anemia & $3(33.3)$ & $2(22.2)$ & $4(44.5)$ & $0(0.0)$ & $0(0.0)$ \\
\hline Thrombocytopenia & $9(100)$ & $0(0.0)$ & $0(0.0)$ & $0(0.0)$ & $0(0.0)$ \\
\hline \multicolumn{6}{|l|}{ Non-hematologic } \\
\hline Nausea/vomiting & $9(100)$ & $0(0.0)$ & $0(0.0)$ & $0(0.0)$ & $0(0.0)$ \\
\hline Diarrhea & $5(55.6)$ & $3(33.3)$ & $1(11.1)$ & $0(0.0)$ & $0(0.0)$ \\
\hline Radiation dermatitis & $9(100)$ & $0(0.0)$ & $0(0.0)$ & $0(0.0)$ & $0(0.0)$ \\
\hline
\end{tabular}

CCRT, concurrent chemoradiotherapy; RT, radiotherapy.

rate of $46.3 \%$ (19/41 patients) in patients with uterine cervical carcinoma (11), which was superior to that obtained with cisplatin $(35.9 \%$; 14/39 patients). Regarding adverse drug reactions, although the nephrotoxicity of nedaplatin was confirmed to be milder compared to cisplatin, certain patients developed grade 3 or 4 myelosuppression (33.6 and $31.1 \%$ for thrombocytopenia and leukopenia, respectively). Nedaplatin has been used clinically in Japan as an alternative to cisplatin for patients with cervical carcinoma. Previous studies examining nedaplatin-based CCRT have demonstrated that nedaplatin is an effective and well-tolerated regimen for invasive cervical carcinoma (20-22). However, the use of this agent in the clinical setting of CCRT in patients with cervical carcinoma is limited.

In their collaborative dose escalation study, Hatae et al suggested that CCRT with the administration of nedaplatin at $80 \mathrm{mg} / \mathrm{m}^{2}$ on Days 1 and 29 for cervical carcinoma was safe and effective (18). In this study, the main side effects for the nedaplatin dose of $80 \mathrm{mg} / \mathrm{m}^{2}$ were digestive disorders including nausea and anorexia and bone marrow suppression, such as leukopenia, neutropenia and thrombopenia. The efficacy was partial response (PR) or better in the patients (18). In the present study, a retrospective analysis was carried out to evaluate whether or not CCRT with the administration of nedaplatin at $80 \mathrm{mg} / \mathrm{m}^{2}$ on Days 1 and 29 is safe and superior to RT alone in Japanese patients with advanced stage of cervical carcinoma.

Our retrospective study demonstrated no significant improvement in the initial response, PFS and OS in patients treated with nedaplatin-based CCRT, compared to RT alone. Mainly due to the small number of patients enrolled, as well as the retrospective nature of this study, other potential biases, such as the age of the patients may have influenced the results.

Nedaplatin-based CCRT was well-tolerated in our patient population. Grade 3-4 leukopenia was observed in 3/8 (37.5\%) of patients treated with nedaplatin-based CCRT, which was higher compared to the RT-group. This incidence of grade 3-4 leukopenia was lower compared to CCRT with weekly nedaplatin in advanced uterine cervical carcinoma (44.5-50.0\%) $(21,22)$. One patient treated with nedaplatin-based CCRT required granulocyte colony-stimulating factor (G-CSF) injections for 2 days. No grade 3 or greater non-hematologic toxicity was observed in these treatment groups. Nevertheless, no significant differences were observed in the length of radiotherapy in these treatment groups, suggesting that nedaplatin-based CCRT using the present regimen was clinically well-tolerated.

As shown in Table III, the difference in the failure patterns was not statistically significant $(\mathrm{P}=0.1428)$, while nedaplatin-based CCRT resulted in fewer distant relapses in this patient population. However, only one patient had distant recurrence in nedaplatin-based CCRT, the 4 patients with relapse had distant recurrence in the RT group. This finding suggests that nedaplatin-based CCRT may control distant relapses.

Nevertheless, the statistical power of this study is limited due to several reasons, such as the relatively small cohort of patients, the heterogeneity of the patient population, as well 
as its retrospective nature. Consequently, additional studies conducted on a mature population are strongly recommended.

\section{References}

1. Goonatillake S, Khong R and Hoskin P: Chemoradiation in gynaecological cancer. Clin Oncol (R Coll Radiol) 21: 566-572, 2009.

2. Pearcey R, Brundage M, Drouin P, et al: Phase III trial comparing radical radiotherapy with and without cisplatin chemotherapy in patients with advanced squamous cell cancer of the cervix. J Clin Oncol 20: 966-972, 2002.

3. Tzioras S, Pavlidis N, Paraskevaidis E and Ioannidis JP: Effects of different chemotherapy regimens on survival for advanced cervical cancer: systematic review and meta-analysis. Cancer Treat Rev 33: 24-38, 2007.

4. Keys HM, Bundy BN, Stehman FB, et al: Cisplatin, radiation, and adjuvant hysterectomy compared with radiation and adjuvant hysterectomy for bulky stage IB cervical carcinoma. N Engl J Med 340: 1154-1161, 1999.

5. Morris M, Eifel PJ, Lu J, et al: Pelvic radiation with concurrent chemotherapy compared with pelvic and para-aortic radiation for high-risk cervical cancer. N Engl J Med 340: 1137-1143, 1999.

6. Rose PG, Bundy BN, Watkins EB, et al: Concurrent cisplatinbased radiotherapy and chemotherapy for locally advanced cervical cancer. N Engl J Med 340: 1144-1153, 1999.

7. Whitney CW, Sause W, Bundy BN, et al: Randomized comparison of fluorouracil plus cisplatin versus hydroxyurea as an adjunct to radiation therapy in stage IIB-IVA carcinoma of the cervix with negative para-aortic lymph nodes: a gynecologic oncology group and southwest oncology group study. J Clin Oncol 17: 1339-1348, 1999.

8. Peters WA III, Liu PY, Barrett RJ II, et al: Concurrent chemotherapy and pelvic radiation therapy compared with pelvic radiation therapy alone as adjuvant therapy after radical surgery in high-risk early-stage cancer of the cervix. J Clin Oncol 18 : 1606-1613, 2000.

9. Fukuda M, Shinkai T, Eguchi K, et al: Phase II study of (glycolate-O,O') diammineplatinum(II), a novel platinum complex, in the treatment of non-small cell lung cancer. Cancer Chemother Pharmacol 26: 393-396, 1990.

10. Inuyama Y, Miyake H, Horiuchi M, Hayasaki K, Komiyama S and Ota K: [A late phase II clinical study of cis-diammine glycolato platinum, 254-S, for head and neck cancers]. Gan To Kagaku Ryoho 19: 871-877, 1992.
11. Kato T, Nishimura H, Yakushiji M, et al: [Phase II study of 254-S (cis-diammine glycolato platinum) for gynecological cancer]. Gan To Kagaku Ryoho 19: 695-701, 1992.

12. Uehara T, Watanabe H, Itoh F, et al: Nephrotoxicity of a novel antineoplastic platinum complex, nedaplatin: a comparative study with cisplatin in rats. Arch Toxicol 79: 451-460, 2005.

13. Kawai Y, Taniuchi S, Okahara S, Nakamura M and Gemba M: Relationship between cisplatin or nedaplatin-induced nephrotoxicity and renal accumulation. Biol Pharm Bull 28: 1385-1388, 2005.

14. Kameyama Y, Okazaki N,Nakagawa M,Koshida H, Nakamura M and Gemba M: Nephrotoxicity of a new platinum compound, 254-S, evaluated with rat kidney cortical slices. Toxicol Lett 52: 15-24, 1990.

15. Sasaki Y, Amano T, Morita M, et al: Phase I study and pharmacological analysis of cis-diammine(glycolato)platinum (254-S; NSC 375101D) administered by 5-day continuous intravenous infusion. Cancer Res 51: 1472-1477, 1991.

16. Nakamura Y, Hasegawa M, Hayakawa K, et al: Induction of p53-dependent apoptosis in vivo by nedaplatin and ionizing radiation. Oncol Rep 7: 261-265, 2000.

17. Tanaka T, Yukawa K and Umesaki N: Radiation reduces carboplatin sensitivity and enhances nedaplatin sensitivity in cervical squamous cell carcinoma in vitro. Eur J Gynaecol Oncol 28: 352-355, 2007.

18. Hatae M, Takahashi T, Kodama S, et al: [A dose escalation study of concurrent chemoradiation therapy with nedaplatin for cervical cancer]. Gan To Kagaku Ryoho 32: 473-478, 2005.

19. Ota K, Wakui A, Majima H, et al: [Phase I study of a new platinum complex 254-S, cis-diammine (glycolato)-platinum (II)]. Gan To Kagaku Ryoho 19: 855-861, 1992.

20. Mabuchi S, Ugaki H, Isohashi F, et al: Concurrent weekly nedaplatin, external beam radiotherapy and high-dose-rate brachytherapy in patients with FIGO stage IIIb cervical cancer: a comparison with a cohort treated by radiotherapy alone. Gynecol Obstet Invest 69: 224-232, 2010

21. Yokoyama Y, Takano T, Nakahara K, et al: A phase II multicenter trial of concurrent chemoradiotherapy with weekly nedaplatin in advanced uterine cervical carcinoma: Tohoku Gynecologic Cancer Unit Study. Oncol Rep 19: 1551-1556, 2008.

22. Niibe Y, Tsunoda S, Jobo T, et al: Phase II study of radiation therapy combined with weekly nedaplatin in locally advanced uterine cervical carcinoma (LAUCC): kitasato gynecologic radiation oncology group (KGROG 0501)-initial analysis. Eur J Gynaecol Oncol 29: 222-224, 2008. 